Marsson, Beitrag zu, Kenntniss der Eisenweinsteine. 169

\title{
Beitrag zur Kenntniss der Eisenweinsteine;
}

\author{
von \\ Th. Marsson, \\ A potheker in Wolgast.
}

Die ältere wiirtembergische Pharmakopöe von 1771, ein für ihre Zeit treffliches Werk, hatte zwei Eisenweinsteine, die globuli martiales und den Tartarus chalybeatus. Das 10 Jahre später erschienene Dispensatorium BorussoErandenburgicum nalım nur das erste Präparat, die globuli martiales auf, ebenso die zweite und dritle Auflage der Pharmacopoea borussica. Erst in der vierten Auflage erscheint wieder der Tartarus chalybealus als $T$. ferruginosus seu martiatus in veränderter Vorschrift, und in die kaum verschiedene fünfte Aullage ging diese Vorschrift mit über, doch gehört dies Präparat zu der Abtheilung der "medicamenta, quae in offucinis praesto esse non debent." Die neue sechste Auflage folgt wieder der dritten und lässt es weg, wofür die Herausgeber der Pharmakopöe den Dank des medicinischen Publicums verdienen, weil eines Theils doch noch eine hinreichende Menge ebenso wirksamer Eisenpräparate in den Apotheken vorräthig ist, andern Theils dieser Tart. ferrugin. zu den Arzneimitteln gehört, die nie von derselben Zusammensetzung darzustellen sind. Auch bat $\mathrm{Sch}$ ach $\mathrm{t}$ es nicht einmal in den zur ncuen Pharmakopöe erschienenen Appendix ${ }^{*}$ ) aufgenommen.

Die globuli tartari ferruginosi sind also officinell geblieben und zwar unter dem neuen Namen: Ferro-Kali tartaricum.

Die Yorschrift zur Bercitung derselben ist in der neuen Pharmakopöe nicht geändert, nur soll die Digestion jetzt so lange fortgesetzt werden, bis eine Probe mit grünlichschwarzer Farbe aufgelöst wird, während früher die Auflösung eine grülich - gelbe Farbe haben sollte. Auch

*) Praeparata chemica et pharmaca composila in pharmacopoeae borussicae editionem sextam non recepta curavit $S$. E. Schacht. 
ist es dem Apotheker erlassen, die Masse in Kugel zu formen, sie soll jetzt ausgetrocknet und zu einem groben Pulver gebracht werden.

Was diese Vorschrift anbetrifft, so ist es die urspriungliche von $\mathrm{Me} \mathrm{yer*}$ ) und $\mathrm{Buchol} \mathrm{z}^{* *}$ ) herrührende, welche beide im Jahre 1811 das Verhältniss der Eisenfeile zum Weinstein wie $1 \mathrm{zu}$ \& feststellten, welches Verhältniss auch schon die dritte Auliage der Pharmacop. boruss. von 1813 aufgenommen hatte.

Dass die neue Pharmakopöe, die so sehr für die Reinheit der Arzneimittel besorgt ist, noch den Tartarus crudus in der Vorschrift gelassen hat, dürfte kaum Billigung finden. Der rohe Weinstein ist ein zu unreines Product, enthält gewöhnlich 15 bis 16 Proc. weinsauren Kalk, ausserdem Sand, Thon, hefige Theile und Pllanzenreste. Dennoch verlangt die Pharmakopöe, dass die Digestion so lange fortgesetzt werde, bis eine Probe sich mit grülich-schwarzer Farbe auflöst. Dies ist aber selbst bei Anwendung eines chemisch reinen Weinsteins nicht zu erreichen, und die Stahlkugeln dürften sehr gut zu nennen sein, wenn sie nur die Hälfte lösliche Bestandtheile enthielten.

Seit Bucholz sind wohl keine Versuche uber Bereitung der Stahlkugel angestellt, welche die Ermittelung des Verhältnisses zwischen Eisen und Weinstein zum Zwecke gehabt hätten. Nur hat Wittstein***) in aller neuester Zeit eine ausfuhrliche Arbeit über die Zusammensetzung der Eisenweinsteine bekannt gemacht, wobei er aus der gefundenen Zusammensetzung die Quantität/Eisen berechnen will, die eigentlich erforderlich ist, um allen Weinstein in Stahlkugel zu verwandeln. Er ist hierbei zu dem Schlusse gekommen, dass wenn Eisen mit Weinstein und Wasser in Berührung gesetzt wird, sich 1 Aeq. Eisen auf Kosten des Wassers oxydirt und mit dem zweiten Aeq. Weinsteinsäure des Weinsteins verbindet, worauf dann ein

\footnotetext{
*) M e yer, J. C. F., Berliner Jahrbuch 1811. S. 108

B*) Buch olz-Tromms dorff's Journal. XXI. S. 40.

3**) Buch ner's Repertorium B. 87. S. 145.
} 
Theil Oxydul höher oxydirt wird, und mit dem entstandenen neutralen weinsteinsauren Kali in der Auflösung bleibt. Der grösste Theil des weinsteinsauren Eisenoxyduls soll sich als unlösliches Pulver abscheiden.

Man bedarf nach dieser Voraussetzung auf 1 Theil Eisen nicht, wie bisher allgemein angenommen wurde, 4 Theile, sondern 7 Theile oder genauer 6,7 Theile Weinstein. Befolgt man das bisherige Verhältniss, so verwandelt sich das überschissige Eisen theilweise in Aethiops und der Rest desselben bleibt dem Präparate metallisch beigemengt.

Ich kann Wittstein's Schliusse nicht für richtig erkennen. Es ist überhaupt sehr zu bedauern, dass Wittstein bei seiner Arbeit, die den ersten Augenblick so gründlich und erschöpfend scheint, nicht mebr Rücksicht auf Analysen der Producte verschiedener Darstellungen genommen hat, er würde dann zu ganz andern Resultaten gelangt sein. So haben seine Formeln durchaus keinen Werth. Wir haben überhaupt bei Körpern, die nicht krystallisiren, kein anderes Kriterium ibrer Reinheit, als wenn die zu verschiedenen Malen dargestellte Verbindung dieselbe $\mathrm{Zu}$ sammensetzung zeigt. Wenn aber ein nicht krystallisirbarer Körper zu verschiedenen Zeiten auf dieselbe Weise dargestellt $\mathrm{nich} \mathrm{t}$ dieselbe Zusammensetzung zeigt, so haben wir allen Grund anzunehmen, dass keine Verbindung von bestimmter Zusammenselzung existirt, oder dass wir es mit untrennbaren Gemengen bestimmter Verbindungen zu thun haben, oder dass Zersetzungen dabei vorkommen, die der Arbeiter nicht bewältigen kann. Letztere beiden Fälle dürften Anwendung auf die Eisenweinsteine finden.

Wir wollen nun zuerst die Stahlkugel in Betracht ziehen und dann zu dem mit Eisenoxyd bereiteten Eisenweinstein übergehen.

Die erste Frage, die bei der Bereitung der Stahlkugel aufgeworfen werden kann, ist:

Wie viel Eisen ist erforderlich, um eine bestimmte Quantität Weinstein in Eisenweinstein zu verwandeln. 
Die zweite Frage:

Welchen Einfluss hat die Temperatur und die Quantität des angewandten Wassers auf die Beschleunigung der Arbeit und die Güte des Präparats?

Es lassen sich diese beiden Fragen bei den Versuchen zusammenfassen.

Vor allen Dingen schien es nöthig zu sein, durch directe Wägung zu ermitteln, wie viel Eisen von einer bestimmten Quantität Weinstein aufgelöst werde. Zu diesen Versuchen lässt sich nun keine Eisenfeile anwenden weil es schwer hält, sich eine von allen Unreinigkeiten freie Feile zu verschaffen, und dann, weil die Stahlkugeln selbst im reinsten Zustande nicht vollständig in Wasser löslich sind, um die zurückbleibende Eisenfeile wägen und in Abzug bringen zu können. Ich bediente mich dazu eines dünnen blanken Claviersaiten-Draths, der spiralförmig zusammengewunden mit dem Weinstein und Wasser in Berübrung gebracht wurde, leicht abgespült und gewogen werden konnte Er darf natürlich nicht zu dün gewählt werden, weil er sonst leicht in mehrere Stücke zerfält, die nicht so gut von der unlöslichen Verbindung der Stahlkugeln getrennt werden können. Auf der andern Seite bietet aber ein dünner Drath wieder mehr Beruhbungspuncte dar, und verdient daher den Vorzug. Ich liess ihn daher nur so lange in Beruhrung als er noch festen Zusammenhang hatte und ersetzte ihn dann durch neuen. $Z u$ den Versuchen warde nun ein völlig chemisch reiner Weinstein angewandt, der aus dem Weinstein des Handels dadurch bereitet war, dass das gepulverte Salz wiederholt mit verdünnter reiner Salzsäure digerirt. und so lange ausgewaschen wurde, bis Silbersalz keine Reaction auf Salzsäure mehr ergab. Das übrig bleibende Salzpulver wurde zum Ueberfluss noch aus heissem Wasser umkrystallisirt und die Krystalle vollständig getrocknet. Der Weinstein war völlig kalkfrei.

Zur Bestimmung der Quantität Eisen, welche vom Weinstein aufyelöst werden kann, wurden folgende Versuche angestellt: 
I. 5,038 Grm. Weinstein wurden mit wenig Wasser und Eisendrath bei gelinder Wärme in Beruhrung gebracht, das verdunstete Wasser stets ersetzt und dabei oft umgerührt. Die Temperatur stieg bisweilen bis nahe zum Kochpuncte des Wassers.

Der Eisendrath hatte an Gewicht verloren:

Nach 8 Tagen ...... $0,6885 \mathrm{Grm}$.

"weitern 4 Tagen. 0,2460

" 3 Tagen ...... 0,0330 "Nun fand weiter kein Verlust statt.

Total-Verlust .. $\overline{0,9675 \mathrm{Grm} .}$ Eisen.

II. 4,569 Grm. Weinstein gleich anfangs mit vielem Wasser und Eisendrath im Sandbade bei sehr ungleicher Temperatur behandelt. Wenn das Wasser ganz verdunstet war, wurde wieder stark verdunnt.

Nach 25 Tagen fand weiter kein Verlust statt.

Der Drath hatte verloren 0,641 Grm. Eisen.

III. 5,336 Grm. Weinstein bei gewöhnlicher Lufttemperatur zwischen $16^{\circ}-28^{\circ} \mathrm{C}$. breiförmig mit Eisendrath in Berührung gesetzt, erst gegen das Ende der Operation bei erhöhter Temperatur digerirt, die den Kochpunct des Wassers nicht ganz erreichte.

Verlust des Eisendraths:

Nacb 18 Tagen........ 1,0185 Grm.

" weitern 7 Tagen.. 0,0560 "Dann kein Verlust mehr.

Total-Verlust. . 1,0745 Grm. Eisen.

IV. 4,121 Grm. Weinstein breiförmig mit Eisendrath warm digerirt, gab folgendes Resultat:

Verlust des Eisens:

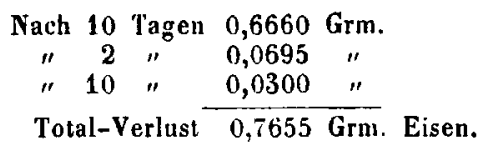

V. 4,203 Grm. Weinstein breiförmig mit Eisendrath bei gewöhnlicher Lufttemperatur zwischen $16^{\circ}-28^{\circ} \mathrm{C}$. in Beruihrung gelassen, erst zuletzt bei allmälig gesteigerter Wärme digerirt, gab als Resultat:

Verlust des Eisens in 10 Tagen..... 0,4935 Grm.

$"$ " " $" \quad$ in weitern 9 Tagen. 0,2070 Tagen....... 0,1900 "Weiter kein Verlust.

Tolal-Verlust $0,8905 \mathrm{Grm}$. Eisen. 
VI. 4,9705 Grm. Weinstein mit Wasser zum dünnen Brei angerührt, mit Eisendrath zwischen $20^{\circ}$ und $50^{\circ} \mathrm{C}$. digerirt.

Verlust an Eisen:

$$
\begin{aligned}
& \text { Nach } 12 \text { Tagen } 0,2215 \mathrm{Grm} . \\
& " 10 " 0,5235 " \\
& " 12 "-\frac{0,2960 "}{120} \\
& \text { Total-Verlust } 1,0410 \mathrm{Grm} \text {. Eisen. }
\end{aligned}
$$

Berechnet man die Quantitäten Eisen, die in diesen 6 Versuchen von 100 Theilen Weinstein aufgelöst wurden, so erhält man folgende Zahlen:

$$
\begin{array}{cccccc}
\text { I. } & \text { II. } & \text { III. } & \text { IV. } & \text { V. } & \text { VI. } \\
19,20 & 14,02 & 20,13 & 18,57 & 21,11 & 20,94 \\
\text { Proc. Eisen. }
\end{array}
$$

Die grosse Verschiedenheit dieser Zahlen, die eine Differenz in Nr. II. und V. von 7,09 Proc. zeigen, scheint den ersten Augenblick unerklärlich, doch werden wir im Verlaufe der Untersuchung die Ursache auffinden.

Wir sehen zu gleicher Zeit hieraus, dass das Verhältniss der Pharmakopöe, 1 Theil Eisen auf 4 Theile Weinstein, kein richtiges ist, es ist mehr Eisen in diesem Verhälıniss, als aufgelöst werden kann. 1 Theil Eisen auf 5 Theile Weinstein würde am besten zutreffen, besonders wenn man bedenkt, dass man bei Anwendung eines rohen Weinsteins, ja selbst noch eines Cremor tartari, Sand, Thon und weinsteinsauren Kalk in Abzug bringen muss, wenn auch der weinsteinsaure Kalk vielleicht eine ähnliche Verbindung mit Eisen bildet. Das nach der Pharmakopöe dargestellte Präparat muss demnach stets noch eine Quantität metallisches Eisen oder Aethiops, worin es durch Oxydation unter Vermittelung des Wassers übergegangen ist, enthalten.

Nach den so eben erhaltenen Resultaten liess sich wohl annehmen, dass die Ausbeute an Eisenweinstein gleichfalls in einem gewissen Verhältnisse zur Temperatur und Quantität des aufgelösten Eisens stände, und desshalb wurde die Ausbeute in diesen 6 Versuchen genau bestimmt. Ich muss hierbei bemerken, dass die Eisenweinsteinmasse, die aus einer leichtlöslichen und einer unlöslichen 
Verbindung besteht, nur sehr schwierig auszutrocknen ist. Die im Wasserbade völlig eingedampfte Flüssigkeit wurde im Trocken-Apparat bei $100^{\circ} \mathrm{C}$. so lange erhitzt, bis zwei Wägungen übereinstimmten, wozu gewöhnlich ein Austrocknen von 6 bis 10 Stunden erforderlich war. Die Wägungen geschahen immer in dem mit einem Uhrgläschen bedeckten Schälchen, weil sonst die Masse schon auf der Waage Feuchtigkeit anzieht und schwerer wird.

Es wurden folgende Resultate erhalten:

I. 5,038 Grm. Weinstein gaben 5,101 $\mathrm{Grm}$. roh.Eisenweinst. (Stahlkugel).

II. $4,569 "$ "

III. $5,336 "$ " "

IV. $4,121 "$ "

V. $4,203 " \prime \prime$

" 4,043 " $"$
" 5,716 "
" 4,109 "
" 4,534 "
" 5,261 "

$\begin{array}{ll}" & " \\ " & " \\ " & " \\ " & "\end{array}$

Auf 100 Theile Weinstein berechnet wirde die Ausbeute gewesen sein:

$\begin{array}{rrr}\text { I. } & 101,25 & \text { Proc. } \\ \text { II. } & 88,49 & \text { " } \\ \text { III. } & 107,12 & " \\ \text { IV. } & 99,70 & " \\ \text { V. } & 107,87 & " \\ \text { VI. } & 105,84 & \end{array}$

Diese Zahlen zeigen deutlich, dass auch die Ausbeute zu dem aufgelösten Eisen in einem gewissen Verhältnisse steht, aber zu gleicher Zeit erkennen wir auch das merkwürdige Resultat, dass die Ausbeute in keinem der 6 Versuche gleich ist der Summe des angewandten Weinsteins und Eisens. Wenn wir auch das basische Wasser, welches der Weinstein noch in dem zweiten Atome Weinsteinsäure enthält und 4,76 Proc. beträgt, abrechnen, so nimmt das Eisen wieder Sauerstoff auf, um als Oxydoxydul die Stelle des Wassers zu vertreten. Denken wir uns nur das Eisen als Oxydul im Eisenweinstein, so müssten z. B., wenn wir Versuch Nr. V. zu Grunde legen, die aufgelösten 21 Proc. Eisen 6,5 Sauerstoff aufnehmen und es wird mehr aufgenommen, weil die Verbindung auch Oxyd enthält, mithin ist die Quantität des aufgenommenen Sauerstoffs noch grösser, als die Menge des ausgeschiedenen basischen 
Wassers. Statt also in diesem Beispiele mindestens 121 Proc. Ausbeute zu erhalten, betrug dieselbe nur 107,87 Proc., es hatle also ein Verlust von ungefahr 14 Proc. statt gefunden. Noch viel auffallender stellt sich dieser Verlust bei den übrigen Versuchen. So wurde in Nr. II. aus 88,49 Proc. Ausbeute erhalien, also ungefahr 26 Proc. weniger als das Gewicht der dazu angewandten Substanzen. Ich darf wohl kaum bemerken, dass alle diese Versuche mit der Vorsicht angestellt wurden, dass ein Verlust durch Ausschülten, Ueberspritzen u. s. w. nicht möglich war. Wir sind daher genöthigt den Verlust auf eine Zersetzung der Weinsteinsäure zu schieben, die theilweise eine andere elementare Zusammensetzung angenommen haben muss und zwar so, dass gewisse Elemente sich zu fluichtigen Producten vereinigt haben. Diese Zersetzung steht nun mit der Temperatur in einem Verhältnisse. Wurde gleich im Anfange eine höhere Temperatur und grössere Quantitàt Wasser angewandt, so trat die Zersetzung fruiher ein, und je fruher dies geschah, um so weniger Weinsteinsäure konnte auflösend auf das Eisen wirken. Diese Zersetzung ganz zu vermeiden, ist nicht möglich, doch wird sie sehr vermindert, wenn im Anfange die Digestion nur bei gewöhnlicher Luftemperatur vorgenommen wird, wie wir aus den Versuchen III., V. und VI. gesehen haben.

Wie ich schon andeutete, besteht der Eisenweinstein aus zwei Verbindungen, einer sehr leicht schon in kaltem Wasser mil grünlich braunschwarzer Farbe löslichen und einer unlöslichen Verbindung. Es schien von Interesse zu erfahren, in welchem Verhältnisse beide Verbindungen in der rohen Stahlkugelmasse zu einander stehen, und ob die verschiedene Behandlung bei der Darstellung einen Einfluss darauf ausübt.

Es wurde zu diesem $Z$ wecke die rohe Kugelmasse in 5 Versuchen so lange mit kaltem Wasser ausgelaugt, als das Wasser noch merklich gefärbt ablief, das Filtrat im Wasserbade abgedampft, und bei $100^{\circ} \mathrm{C}$. ausgetrocknet, bis zwei Wägungen übereinstimmten. Eine absolut genaue 
Trennung beider Verbindungen ist nicht möglich, weil die unlösliche Verbindung bei Berührung der Luft etwas löslich wird, und das Waschwasser, was schon farblos ablief, sich wieder zu färben beginnt. Die lösliche Verbindung bildet in Wasser eine tief grünlich braunschwarze Lösung, und liefert zur Trockne gebracht, eine glänzende, fast schwarze, harzähnliche Masse, deren Pulver eine braungrünliche Farbe besitzt.

II. 4,043 Grm. Stahlkugel gaben 2,180 Grm. lösliche Verbindung.

III. 5,716 "

IV. $4,109 "$

V. 4,534"

$\begin{array}{lllll}" & 2,181 & \text { " } & \text { " } \\ " & 2,692 & \text { " } & \text { " } \\ \text { " } & 2,923 \text { " } & \text { " } & \text { " } \\ \text { " } & 3,074 \text { " } & \text { " }\end{array}$

VI. $5,261 "$

Hiernach enthalten 100 Theile Stahlkugelmasse an löslicher Verbindung beide bei $100^{\circ} \mathrm{C}$. getrocknet:
II. 53,92 Proc.
III. 51,71 "
IV. $65,51 "$
V. $64,48 "$
VI. $58,43 "$

Diese Ausbeuten stimmen, wie die Zahlen anzeigen nicht mil der Quantität des aufgelösten Eisens überein, und es ist schwer zu sagen, warum in Nr. IV., wo nur 18,57 Proc. Eisen aufgelöst wurden, gerade am meisten von der löslichen Verbindung erhalten wurde. kugel.

Wenden wir uns nun zur Zusammensetzung der Stahl-

\section{1) Unlösliche Verbindung.}

Wenn Weinstein mit Eisen und Wasser in Beruhrung gebracht wird, so findet unter Wasserstoffgas-Entwickelung eine Einwirkung statt, das Eisen wird auf Kosten des Wassers oxydirt, und es beginnt eine Abscheidung eines weisslichen Pulvers, das gewöhnlich für weinsteinsaures Eisenoxydul gehalten wird, aber eben so gut eine DoppelVerbindung von weinsteinsaurem Eisenoxydul und weinsteinsaurem Kali sein kann. Diese Verbindung ist vom Weinsteinpulver kaum zu trennen, und wegen seiner leichten Oxydirbarkeit an der Luft, wodurch zu gleicher Zeit

Arch.d. Pharm. CHI. Bds. 2. Hft. 
eine lösliche Verbindung entsteht, nicht gut zu analysiren. Bei längerer Einwirkung der Luft geht nun ein Theil der abgeschiedenen weissen weinsteinsauren Eisenverbindung wieder in die lösliche Eisenweinstein-Verbindung über, und färbt sich dabei immer dunkler grülichbraun. Gleichzeitig beginnt aber auch die Abscheidung eines käsigen, dem Chlorsilber nicht unähnlichen, weissgrünlich gefärbten Niederschlags, der sich bis zur Beendigung des Processes zu bilden scheint. Ausgewaschen sieht, dieser Niederschlag grünlichgrau aus, wird aber beim Trocknen gelblich, offenbar ein Zeichen höherer Oxydation und dadurch etwas in Wasser mit hellgelblicher Farbe löslich, doch gelingt es nicht durch wiederholtes Abdampfen und Auflösen daraus die lösliche Verbindung der Stahlkugel darzustellen.

lch unterwarf die unlösliche Verbindung von verschiedenen Bereitungen auf folgende Weise der Analyse. Die bei $100^{\circ} \mathrm{C}$. getrocknete Verbindung wurde im Platintiegel verbrannt, bei Luftzutritt bis zur gänzlichen Zerstörung der Kohle geglüht, der Glührückstand dann im Kolben in Salzsäure unter Hinzulügung einiger Tropfen Salpetersäure aufgelöst und aus der Lösung durch Ammoniak das Eisenoxyd gefallt, das vollständig ausgewaschen, geglüht und gewogen wurde. Aus dem zur Trockne abgedampften Waschwasser wurde durch Glühen im Platintiegel der Salmiak verjagt und das Kali als Chlorkalium bestimmt. Auf diese Weise habe ich alle Eisenweinstein-Verbindungen analysirt.

Die bei $100^{\circ} \mathrm{C}$. getrocknete unlösliche Verbindung von den Darstellungen Nr. II., IH., IV. und VI. gab:

II. $0,266 \mathrm{Grm}$. gaben $0,0940 \mathrm{Grm}$. Eisenoxyd u. 0,0925 $\mathrm{Grm}$. Chlorkalium entsprechend 0,0574432 Kali.

III. 1) 0,407 Grm. gaben 0,145 Grm. Eisenoxyd, ferner 0,1225 Grm. Chlorkalium entsprech. 0,077397 Grm. Kali.

2) 0,858 Grm. gaben 0,305 Grm. Eisenoxyd, ferner 0,2625 Grm. Chlorkalium entsprech. 0,1658526 Grm. Kali. 
IV. 0,392 Grm. gaben 0,1365 Grm. Eisenoxyd, ferner $0,1275 \mathrm{Grm}$. Chlorkalium, entsprechend 0,08055703 Grm. Kali.

VI. 1) 0,472 Grm. gaben 0,1765 Eisenoxyd, ferner 0,129 Grm. Chlorkalium, entsprechend 0,0815047 Grm. Kali.

2) 0,715 Grm. gaben $0,268 \mathrm{Grm}$. Eisenoxyd, ferner $0,198 \mathrm{Grm}$. Chlorkalium entsprechend 0,1251002 Grm. Kali.

100 Theile lieferten hiernach:

II.

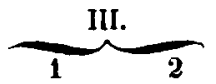

IV.

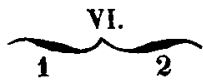

Eisenoxyd $34,39-35,62-35,54-34,82-37,39-37,48$ Proc. Kali ...2 21,59-19,01-19,33-20,55 - 17,26-17,49"

Die Zahlen von den verschiedenen Darstellungen stimmen nun so wenig überein, dass es nicht möglich ist, sich eine Vorstellung von der Zusammensetzung zu machen. Sie zeigen aber auch zu gleicher Zeit, dass eine Verbindung von bestimmter Zusammensetzung gar nicht existirt. Das Eisen dürte in dieser Substanz zum grössten Theil als Oxydul enthalten sein, wovon noch mehr bei der Berührung mit der Luft in Oxyd verwandelt wird. Die Auflösung in Salzsäure giebt mit Kaliumeisencyanür und -Cyanid einen blauen Niederschlag doch mil ersterem am meisten. $O b$ nun noch wirklich die Säure in dieser Verbindung Weinsteinsäure ist, oder ob aus derselben theilweise eine andere entstanden ist, die als solche in dem Körper enthalten ist, muss dahin gestellt bleiben, da es nicht gelingt in zwei Bereitungsarten dieselbe Verbindung von derselben Zusammensetzung zu erhalten.

\section{2) Lösliche Verbindung.}

Es war im Voraus zu vermuthen, dass die lösliche Verbindung der Stahlkugel keine constante Zusammensetzung besitze, wenn man bedenkl, dass bei derselben Quantität Kali in der rohen Stahlkugelmasse das eine Mal 18 Proc., das andere Mal 21 Proc. Eisen enthalten sein konnte. Dass die Analyse daher kein Ergebniss haben würde, welches zur Aufstellung einer Formel füh- 
ren könnte, war als bestimmt anzunehmen. Die Formel, welche Wittstein aufgestellı hat, kann wohl zufälig als der Ausdruck der Analyse des gerade von ihm analysirten Körpers betrachtet werden, wenngleich der Eisengehalt im Verhältniss zu dem von mir erhaltenen Resultat so gering ist, dass man glauben könnte, die so sehr hygroskopische Masse sei vor der Analyse nicht vollständig getrocknet worden. $\mathrm{Zu}$ einem allgemeinen Schlusse berechtigt Wittstein's Formel keinesweges, da man es bei diesem Präparate fortwährend mit Zersetzungsproducten zu thun hat, und doch die Zersetzung nur die Weinsteinsäure allein treffen kann. Wittstein's Formeln gehen aber alle von der Voraussetzung aus, dass die Weinsteinsäure noch unzersetzl in der Verbindung enthalten ist.

Wenn man die rohe Stahlkugelmasse mit kaltem Wasser auslaugt, die Lösung filtrirt und im Wasserbade zur Trockne eindampft, so löst sie sich nicht mebr klar in Wasser auf. Dampft man die vom Bodensatze abfiltrirte Lösung wieder im Wasserbade zur Trockne ein, so findet beim Auflösen in Wasser, wenn man die Lösung die Nacht über stehen lässt, wieder die Abscheidung eines Niederschlages statt, und es ist ein vier- bis fünfmaliges Auflösen erforderlich, bis die Lösung klar bleibt, und beim 24stiundigen Stehen nichts absetzt. Diese Bemerkung hat Witts te in auch schon gemacht, und er erklärt den Niederschlag für weinsteinsaures Eisenoxyduloxyd, welches durch das wiederholte Abdampfen und Auflösen ausgeschieden wird. Jedenfalls beruht diese Abscheidung auf einer Zersetzung, denn der abgeschiedene Niederschlag löst sich in Wasser fast gar nicht auf, und musste also in einer andern Verbindung in dem löslichen Theile der Stahlkugel enthalten sein.

Die zur Analyse angewandte Substanz war durch so häufiges Auflösen, bis die Lösung klar blieb, erhalten und wurde stets bei $100^{\circ} \mathrm{C}$. so lange getrocknet, bis zwei Wägungen übereinstimmten. 


\section{Bereitung:}

Nro.IV. 1) 0,7065 Grm. gaben 0,163 Grm. Eisenoxyd, ferner $0,340 \mathrm{Grm}$. Chlorkalium entsprechend 0,214818 Kali.

2) 0,5525 Grm. gaben 0,1275 Grm. Eisenoxyd ferner 0,2655: Grm. Chlorkalium entsprechend $0,16774809 \mathrm{Grm}$. Kali.

Nro. V. 0,756 Grm. gaben 0,192 Grm. Eisenoxyd, ferner $0,348 \mathrm{Grm}$. Chlorkalium entsprechend 0,21987335 Grm. Kali.

Nro. VI. 0,8975 Grm. gaben 0,233 Grm. Eisenoxyd, ferner $0,4.195$ Grm. Chlorkal., entsprechend 0,26504847 Grm. Kali.

Auf 100 Theile berechnet giebt diess:

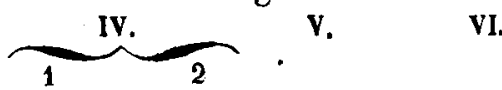

Eisenoxyd 23,07 - 23,06 - 25,39-25,96 Proc.

Kali... 30,40 - 30,36 - 29,08 - 29,53 "

Bei der Bereitung dieser Präparate wurde in

Nro. IV. V. VI.

$18,5721,11 \quad 20,94$ Proc. Eisen

aufgelöst. Mit diesem aufgelösten Eisen steht auch die Zusammensetzung der löslichen Verbindung in Verhältniss, wenngleich der Unterschied in der Zusammensetzung nicht so sehr verschieden ist, wie bei der unlöslichen Verbindung. In Nro. IV., wo das wenigste Eisen aufgelöst wurde, ist der Kaligehalt am grössten und der Eisengehalt am kleinsten, in V. und VI stimmen die Analysen so ziemlich genau überein, dass man beide gleich zusammengeselzt betrachten kann, aber die Quantität des aufgelösten Eisens war auch fast gleich, da eine Differenz von 0,17 Proc. Eisen, welches Nro. V. mehr enthielt, in die Grenze der Beobachtungsfehler fältt. Doch, glaube ich, berechtigt dies ubereinstimmende Resultat keineswegs zu der Annahme, dass hierdurch die wahre Zusammensetzung der löslichen Stahlkugelmasse gefunden werden könne. Wie wir schon früher gesehen haben, fand bei der Bereitung ein Verlust von mindestens 14 Proc. statt, der auf Zersetzung der Weinsteinsäure geschoben werden musste, 
und es wäre vielleicht möglich, durch besondere Vorsichtsmaassregeln dahin zu gelangen, noch eine grössere Quantität Eisen aufzulösen, aber dann würde die Analyse wieder andere Resultate geben.

Dass die Verbindung gleichzeitig Oxyd und Oxydul enthält, ist bekannt. Ich habe es für überflüssig gehalten, das Verbältniss beider zu einander zu bestimmen, da das Resultat doch nicht übereinstimmend ausfallen konnte.

Werfen wir nun noch am Schlusse die Frage auf, wie viel Eisen 1 Aeq. Weinstein aufzulösen im Stande wäre, so ist diese Frage allerdings wegen der dabei statt findenden Zersetzung der Weinsteinsäure nicht mit Gewissheit zu beantworten, doch scheint das Verhältniss 2 Aeq. Weinstein auf 3 Aeq. Eisen der Wahrheit am nächsten zu kommen. Es würden dann 100 Theile Weinstein 22,3 Theile Eisen erfordern, das Maximum in den Versuchen war 21,11.

II. Der mit Eisenoxyd bereitete Eisenweinstein.

Zum innerlichen Gebrauche hatte die preussische Pharmakopöe bis jetzt unter dem Namen Tartarus ferruginosus seu martiatus ein Präparat, welches aus Eisenoxydhydrat und Cremor Tartari dargestellt wurde. Es sollten $\uparrow_{\frac{1}{2}}$ Unzen der officinellen Eisenchloridlösung mit Aetzkali gefällt werden, das sorgfältig ausgesüsste Eisenoxydhydrat mit einer Unze gereinigten Weinstein und 8 Unzen Wasser bis zur Hälfte gekocht, darauf bei gelindem Feuer bis zum duinnen Extract eingedampft werden. Die erkaltete Masse wurde dann in drei Unzen Wasser aufgelöst und die Lösung zur Trockne abgedampft.

Diese Vorschrift liess manches zu wünschen ubrig; die Fällung des Eisenoxyds durch Kali war nicht zweckmässig, die Eisenchloridlösung enthielt nicht immer dieselbe Quantität Eisen, wesshalb auch die Menge des angewandten Eisenoxydhydrats nicht dieselbe sein konnte.

Jeder, der nach dieser Vorschrift den Tart. ferrug. darstellte, wird bei einiger Aufmerksamkeit die Erfahrung gemacht haben, dass die Quantität Eisenoxydhydrat lange nicht hinreichend ist, um den Weinstein zu sättigen, und 
dass das Wiederautlösen der erkalteten Masse, welches ursprünglich wohl zur Abscheidung des weinsteinsauren Kalks dienen sollte, gleichzeitig auch die Abscheidung des überschüssigen Weinsteins zur Folge hat, der von einem Niederschlage einer weinsteinsauren Eisenverbindung begleitel ist.

Letzteres hat $\mathrm{Dufl}$ os*) schon hinlänglich beobachtet und um diesen Verlust zu vermeiden, die Vorschrift geändert. Er schlägt vor, 6 Theile krystallisirtes kohlensaures Natron in der zehnfachen Menge Wasser zu lösen, und unter Kochen 4 Theile officinelle Eisenchloridlösung hinzuzugiessen. Den ausgewaschenen Niederschlag von Eisenoxydhydrat vermischt er mit $2 \frac{1}{2}$ Theilen gepulverten mit Salzsäure gereinigten Weinsteins und digerirt in gelinder Wärme bis zur Auflösung, dann setzt er noch $4 \frac{1}{4}$ Theile trocknes neutrales weinsteinsaures Kali hinzu und bringt die Flüssigkeit zur Trockne. Nach ihm soll die mit Hülfe des Weinsteins bewirkte Auflösung des Eisenoxydhydrats $\mathrm{KO} \overline{\mathrm{T}}+\mathrm{Fe}^{2} \mathrm{O}^{3} \overline{\mathrm{T}}$ enthalten, verdiunnt man aber die Auflösung mit Wasser und lässt erkalten, so scheidet sich eine grosse Menge des weinsteinsauren Eisenoxyds ab. Trocknet man jetzt die Aullösung ein und löst sie dann wieder in Wasser auf, so scheidet sich abermals ein Antheil Eisenoxydsalz aus und dieses wiederholt sich nach Duflos, bis in der Verbindung das weinsteinsaure Eisenoxyd zum weinsteins. Kali sich verhält wie $\mathrm{Fe}^{2} \mathrm{O}^{3} \overline{\mathrm{T}}: 4 \mathrm{KOT}$.

Auf diese Weise würde also $\frac{3}{4}$ des weinsteinsauren Eisenoxyds verloren gehen, was man vermeidet, wenn der Lösung von vornherein noch 3 Aeq. neutrales weinsteinsaures Kali zugefuigt werden.

Zur Erforschung des besonderen Verhaltens dieses Präparats wurde reines durch Ammoniak gefälltes Eisenoxydhydrat mit einem Ueberschuss von reinem Weinstein und Wasser im Wasserbade erhitzt. Nach Auflösung des Eisenoxyds wurde die Flüssigkeit von dem überschüssigen Weinsteinpulver abfiltrirt. Sie batte eine rein braune

*) Duflos, Apothekerbuch 2. Aufl. Bd. 1. S. 315. 
Farbe, ohne einen Stich ins Grünliche, gab mit Salzsäure bis zur Auflösung des abgeschiedenen basischen weinsteinsauren Eisenoxyds und dann mil Kaliumeisencyanid versetzt eine deutliche Reaction auf Eisenoxydul. Beim Abdampfen nahm sie eine mehr grünliche Farbe an, und trocknete und zu einer glänzenden grünbraunen harzartigen Masse aus, die sich aber nicht mehr klar in Wasser auflöst, sondern ein gelbliches Pulver fallen lässt. Dampft man die abfiltrirte Flüssigkeit zur Trockne ein, so findet beim Auflösen in Wasser wieder eine Abscheidung statt, die besonders hervortritt, wenn man sie 24 Stunden stehen lässt. Aller Wahrscheinlichkeit nach musste diese Abscheidung eine Grenze baben und dann eine Verbindung von bestimmter Zusammensetzung erhalten werden. Ich setzte daher das Auflösen und Abdampfen sech zehn Mal fort, ohne dass sich nun die Verbindung in Wasser klar auflöste, und beim Stehenlassen klar blieb, es fand noch immer eine Abscheidung statt, die um so grösser zu sein schien, je vollständiger die Masse vorher ausgetrocknet war.

Die Verbindung wurde jetzt analysirt.

1) $0,825 \mathrm{Grm}$. Substanz bei $100^{\circ} \mathrm{C}$. getrocknet, gaben $0,211 \mathrm{Grm}$. Eisenoxyd und $0,353 \mathrm{Grm}$. Chlorkalium, entsprechend 0,223032 Kali.

2) $0.672 \mathrm{Grm}$. gaben $0,171 \mathrm{Grm}$. Eisenoxyd und 0,287 Grm. Chlorkalium entsprechend 0,181332 Kali.

Auf 400 Theile berechnet:

$\begin{array}{cc} & 1 \\ \text { Eisenoxyd } 25,57 & 25,44 \\ \text { Kali .... } 27,03 & 26,98 .\end{array}$

Dass die Verbindung auch Oxydul enthält, bemerkte ich schon vorher.

Dulk fand einen Tart. ferrug. der Pharmakopöe zusammengesetzt aus:

$$
\begin{aligned}
& \text { Kali..........31,49 } \\
& \text { Eisenoxyd.....13,06 } \\
& \text { Weinsteinsăure. .55,45 }
\end{aligned}
$$

woraus zu gleicher Zeit ersichtlich ist, dass das Präparat jedesmal von anderer Zusammensetzung erhalten wird 
und eine bestimmte Verbindung sich bei einem Ueberschuss von Weinstein nicht darstellen lässt.

Die Niederschläge, die sich beim Auflösen des Eisenweinsteins abscheiden, scheinen ebenfalls keine bestimmte Zusammensetzung zu haben. Wenn die Niederschläge ausgewaschen, bei $100^{\circ} \mathrm{C}$. getrocknet und im Platintiegel bei Luftzutritt verbrannt wurden, so erhielt ich in 3 Versuchen folgende Glïhriickstände:

38,72 Proc. 58,31 und 63,52 Proc.

Die beiden letzten brausten stark mit Säuren, enthielten also kohlensaures Kali, die Abscheidung besteht daher nicht immer aus reinem weinsteinsaurem Eisenoxydul, sondern enthält auch weinsteinsaures Kali.

Es liess sich aber nun wohl vermuthen, dass man eine Verbindung von bestimmter Zusammensetzung erhalten könne, wenn man Weinsteinlösung mit einem Ueberschuss von Eisenoxydhydrat behandelt. Auch hat Wittstein eine solche Verbindung analysirt und nach ihm soll der mit Ueberschuss von Eisenoxydhydrat bereitete Eisenweinstein folgende Zusammensetzung haben:

$$
\begin{aligned}
& \text { Kali ........19,30 } \\
& \text { Eisenoxydul ... 4, 4, } 4 \\
& \text { Eisenoxyd ....24,37 } \\
& \text { Weinsteinsäure } 52,68
\end{aligned}
$$

woraus er die Formel $4(\mathrm{KO}+\overline{\mathrm{T}})+(\mathrm{FeO}+\overline{\mathrm{T}})+$ $3\left(\mathrm{Fe}^{2} \mathrm{O}^{3}+\overline{\mathrm{T}}\right)$ entwickelt. Wittstein hat auch hier wieder den Fehler begangen, dass er sich nicht überzeugte, ob auch wiederholte Darstellungen dasselbe Präparat geben, er würde dann bald gesehen haben, dass diese Verbindung wohl möglich sei, wie noch viele andere, dass aber der Weinstein eine viel bedeutendere Quantität Eisenoxyd aufzunehmen in Stande sei. Ich stellte dies Präparat zu verschiedenen Malen mit einem Ueberschuss von Eisenoxyd dar, so dass beim Filtriren der Lösung Eisenoxyd auf dem Filter zurückblieb. Da aber die Analyse stets verschiedene Resultate gab, so löste ich den Weinstein im Wasserbade in hinreichender Menge Wasser auf, setzte dann so viel breiförmiges Eisenoxydhydrat hinzu, dass 
das Ganze zu einem dünnen Brei gestand, der unter Umrübren zur Trockne gebracht wurde. Die trockne Masse wurde mit kaltem und heissem Wasser ausgelaugt, fltrirt, und wieder zur Trockne abgedampft. Die harzartige Masse ist glänzend, dunkel schwarzbraun, das Pulver hat eine reine braunrothe, dem reinen Eisenoxyde sehr ähnliche Farbe, ohne einen Stich ins Grünliche, wie beim officinellen Präparate. Die intensiv dunkelrothbraune Auflösung wird weder durch Kaliumeisencyanür noch -Cyanid verändert, setzt man aber vorher Salzsäure hinzu, bis das basische Eisenoxyd wieder aufgelöst ist, so geben beide Reagentien blaue Niederschläge. Die Verbindung enthielt also gleichzeitig Oxydul. Die Reduction des Oxyds zu Oxydul findet aber nicht allein gegen das Ende des Abdampfens statt, sondern schon, wenn die erste Quantität Eisenoxyd vom Weinstein gelöst ist. Bei diesem Präparat findet nun auch eine ganz ähnliche Erscheinung statt, wie beim vorhergehenden. Löst man die Verbindung auf, lässt über Nacht stehen, so findet die Abscheidung eines Niederschlages statt, der ebenfalls eine rein braunrothe Farbe besitzt. Dampft man ab und löst wieder auf, so wiederholt sich diese Abscheidung auch. Nach fünfmaligem Auflösen und Abdampfen zur Trockne konnte noch kein Präparat erhalten werden, was sich vollständig klar in Wasser löste. Hiernach war es auch vorauszusehen, dass die Analyse keine uibereinstimmenden Resultate geben würde.

Bei $100^{\circ} \mathrm{C}$. getrocknet gaben:

I. 0,657 Grm. Substanz 0,2735 Grm. Eisenoxyd und 0,166 Chlorkalium, entsprechend $0,10488 \mathrm{Grm}$. Kali.

II. $0,5595 \mathrm{Grm}$. gaben 0,270 Grm. Eisenoxyd und 0,1165 Grm. Chlorkalium, entsprechend 0,073609 Grm. Kali.

III. $0,6665 \mathrm{Grm}$. gaben 0,3835 Eisenoxyd und 0,133 Grm. Chlorkalium, entsprechend 0,084032 Kali.

IV. 0,595 Grm. gaben 0,342 Grm. Eisenoxyd und 0,105 Grm. Chlorkalium, entsprechend 0,066341 Kali.

V. 0,610 Grm. gaben $0,361 \mathrm{Grm}$. Eisenoxyd und 0,112 Grm. Chlorkalium, entsprechend 0,0707638 Kali. 
Siepell, kleine Mittheilungen.

Auf 100 Theile berechnet giebt dies:

\begin{tabular}{|c|c|c|c|c|}
\hline I. & II. & III. & IV. & V. \\
\hline .... 15,96 & 13,15 & 12,60 & 11,14 & 11,60 . \\
\hline
\end{tabular}

Zu den drei letzten Darstellungen war ein ausserordentlich grosser Ueberschuss von Eisenoxyd angewandt, und sie enthalten auch die grösste Menge davon, aber die Zusammensetzung ist durchaus nicht übereinstimmend.

Man kann daher mit Bestimmtbeit annehmen, dass eine Verbindung von gleicher Zusammensetzung nicht darzustellen ist. Die Gründe dazu sind allerdings nicht leicht zu errathen. Jedenfalls spielt die durch die Weinsteinsäure bewirkte Reduction des Eisenoxyds eine Rolle hierbei, ein Entweichen von Gasarten habe ich dabei aber nicht bemerken können.

\section{Kleine Mittheilungen;}

\section{Dr. Siepell in St. Petersburg.}

Die Pharm. castr. ruthenica giebt unter Polus acidus vegetabilis folgende Vorschrift:

Rec. Decocti Hordei libras duas, Bitartratis Potassae drachmas duas, vel quantum satis ad fluidum saturandum, Syrupi simplicis semiunciam. U. Synodha, synochus, varicella et alii sthenici morbi. Corticis citri medicas additamentum eundem gratiorem facit. Recte tamen huic potui substituitur posca, Ruthenis kbacz (Kwass) dicta.

Es lässt sich darüber Folgendes sagen. Der Kwass wird in russischen Hospitälern als Getränk allgemein gebraucht. Der Kwass ist das älteste Nationalgetränk der Russen und fast ein Jabrtausend ist sein Gebrauch schon bekannt; kein Land hat ein solches Nationalgetränk aufzuweisen, welches in allen Ständen so lange sich in der Mode erhalten hätte. In N es to r's russischen Geschichtsannalen 1767 Seite 89 heisst es, dass unter Wladimir dem Grossen (reg. von 980-1014) bei den Hoffesten an Kwass kein Mangel gewesen sei; Heberstein spricht gleichfalls in seinem rerum Muscoviticarum commentarius 